\title{
Efecto Agudo en la Atención de Niños de 12 a 14 Años de una Clase de Educación Física
}

\author{
Acute Effect on the Attention of Children 12 to 14 Years in a Class of Physical \\ Education
}

\author{
Beatriz Berrios Aguayo ${ }^{1}$, Pedro Ángel Latorre Román ${ }^{2}$, Jesús Salas Sánchez ${ }^{3}$ y Antonio Pantoja \\ Vallejo ${ }^{4}$
}

\section{Resumen}

El propósito de esta investigación fue evaluar el efecto agudo de dos tipos de clases de Educación Física (EF) en la atención selectiva en estudiantes de Educación Secundaria Obligatoria Los participantes fueron 98 estudiantes (edad= $12.96 \pm .96$ años), 47 niñas y 51 niños. Los estudiantes fueron asignados aleatoriamente a los grupos experimentales (GE $n=65)$ o al grupo control $(\mathrm{GC}, \mathrm{n}=33$ ). Un grupo experimental recibió una clase de juegos predeportivos (JPD, n=33) y el otro una clase de juegos aeróbicos de alta intensidad (JA). Se empleó el test D2 para analizar la atención y la concentración. Los GEs mostraron un mejor rendimiento en las respuestas totales contestadas, en la puntuación total $(p<.05)$ y en la concentración $(p<.05)$ que el GC Los resultados demostraron que diferentes clases de EF tienen un efecto agudo beneficioso en la atención selectiva y la concentración en niños de Educación Secundaria

Palabras clave: atención, Educación Física, Educación Secundaria Obligatoria.

\begin{abstract}
The purpose of this research was to evaluate the acute effect of two types of Physical Education (PE) classes in selective attention in Compulsory Secondary Education students. The participants were 98 students (age = $12.96 \pm .96$ years), 47 girls and 51 boys. Students were randomly assigned to the experimental groups (GE $\mathrm{n}=65)$ or the control group $(\mathrm{GC}, \mathrm{n}=33)$. One experimental group received one class of predictive games (JPD, n=33) and another high intensity aerobics class (JA). The D2 test was used to analyze attention and concentration The D2 test was used to analyze attention and concentration. The EGs showed a better performance in the total answers answered, in the total score $(p<.05)$ and in the concentration $(p<.05)$ than the CG. The results showed that different classes of EF have a beneficial acute effect on selective attention and concentration in secondary school.
\end{abstract}

Keywords: attention, physical education, Secondary School

\footnotetext{
${ }^{1}$ Psicopedagogía, Universidad de Jaén, España. Contratada Predoctoral. Tel.: +34665814324. Correo: bberrios@ujaen.es

${ }^{2}$ Ciencias de la Actividad física y el deporte, Universidad de Jaén, España. Profesor Titular. Tel.: 953212710. Correo: platorre@ujaen.es

${ }^{3}$ Ciencias de la Actividad Física y el Deporte, Universidad Autónoma de Chile, Chile. Tel.: +34 659552578. Correo: jsalas@ujaen.es

${ }^{4}$ Pedagogía, Universidad de Jaén, España. Profesor Titular. Tel.: 953 212358. Correo: apantoja@ujaen.es 


\section{Introducción}

La actividad física (AF) proporciona múltiples beneficios para la salud (Ahn \& Fedewa, 2011; Janssen \& Leblanc, 2010). En los últimos años ha habido un creciente interés en determinar qué beneficios cognitivos en el ámbito académico podrían tener las clases de Educación física (EF) o la AF en general (Budde, Voelcker-Rehage, Pietraßyk-Kendziorra, Ribeiro, \& Tidow, 2008; Ellemberg \& St-Louis-Deschênes, 2010; Gallotta et al., 2014; Verburgh, Königs, Scherder \& Oosterlaan, 2014). Gallotta et al., (2014) y Soga, Shishido y Nagatomi (2015) demostraron que la duración del ejercicio, la intensidad de éste, la condición física de los participantes y el tipo específico de ejercicio físico realizado son moderadores importantes en la asociación entre la AF y el rendimiento cognitivo. Es importante señalar que el nivel individual de condición física está potencialmente determinado con la demanda de AF lo que influye en las funciones ejecutivas (Soga, Shishido, \& Nagatomi, 2015).Los niños que están en buena forma física y por lo tanto disponen de un nivel de condición física mayor, presentan una mayor activación cortical que se corresponde con un mayor rendimiento académico (Tomporowski, Davis, Miller, \& Naglieri, 2008). Chaddock, Pontifex, Hillman y Kramer (2011) demostraron la importancia de la AF y la aptitud aeróbica para maximizar la salud del cerebro y la función cognitiva durante el desarrollo infantil. En este sentido, la realización de ejercicios aeróbicos promueve el desarrollo físico e intelectual (Vidarte-Claros, Velez-Álvarez, SandovalCuellar, \& Alfonso-Mora, 2011; Delgado \& Prado-Gascón, 2016).

Por otro lado, tanto los ejercicios de baja como de alta intensidad pueden mejorar el desarrollo de las funciones ejecutivas (Best, 2010). Pesce, Crova, Cereatti, Casella, \& Bellucci (2009) investigaron los efectos del ejercicio físico en el rendimiento de la memoria en niños de 1112 años a través de dos sesiones de EF (un circuito de entrenamiento aeróbico vs juegos de equipo) semejantes en intensidad pero diferentes en cuanto al compromiso cognitivo y social. Los resultados mostraron que el recuerdo inmediato fue mayor después de los juegos de equipo, mientras que el recuerdo tardío fue mayor después de los juegos de equipos así como después del entrenamiento aeróbico. Los efectos diferenciales de los dos tipos de ejercicio en el recuerdo inmediato y tardío indican que la memoria puede ser mejorada no solo mediante activación fisiológica sino también por la actividad cognitiva presente en el ejercicio físico

Igualmente, Pontifex, Hillman, Fernhall, Thompson y Valentini (2009) llegaron a la conclusión de que una serie aguda de ejercicio aeróbico puede facilitar el rendimiento cognitivo. A su vez, Tomporowski (2003) informó de que un ejercicio aeróbico realizado en períodos de hasta 60 minutos facilita aspectos específicos del tratamiento de la información y del rendimiento cognitivo en adultos. De manera semejante, Pérez, Padilla, Parmentier y Andrés (2014) evaluaron el efecto de la práctica deportiva en las redes atencionales de estudiantes universitarios, demostraron un efecto agudo de una sesión de 60 minutos de ejercicio aeróbico en la atención. Por otro lado, Altenburg, Chinapaw y Singh (2016) formaron tres grupos de estudiantes de 10-13 años de edad. El primer grupo no realizaba AF antes ni durante las sesiones académicas, el segundo grupo realizaba AF justo antes de la sesión académica y el último grupo realizaba dicha actividad antes y después de la sesión académica. Los resultados demostraron que el grupo que realizaba $\mathrm{AF}$ antes y después obtuvieron mejores puntuaciones en la evaluación de la atención selectiva. Además, Schmidt, Egger y Conzelmann (2015) revelaron que el rendimiento atencional de niños de 10-11 años aumentó tras 90 minutos de la realización de una clase de EF basada en ejercicios de coordinación.

Una reciente revisión corrobora los hallazgos de gran parte de estos estudios y es que según Maureira-Cid y Flores-Ferro (2017), más del 70\% de los estudios analizados señalan los efectos positivos del ejercicio físico sobre la atención, incluyendo trabajos con aplicaciones de una sesión de entrenamiento $\mathrm{o}$ de varias semanas/meses (Luliano et al., 2015; Vidoni et al., 2015). Por tanto, el ejercicio aeróbico también produce cambios fisiológicos específicos, no sólo generales en el cerebro, además de causar una respuesta inmediata neuroquímica que puede mejorar el rendimiento cognitivo (Best, 2010). En este sentido, se ha demostrado que el ejercicio 
afecta positivamente al rendimiento cognitivo, debido a la conexión neuronal entre el cerebelo y la corteza frontal (Budde et al., 2008).

Sin embargo, algunos trabajos como los de Wilson, Olds, Lushington, Petkov y Dollman (2016) o Dutke, Jaitner, Berse y Barenberg (2014) no mostraron influencia del entrenamiento físico sobre la atención, lo que hace necesario la realizacion de más estudios, como el presente, orientados a determinar las intensidades y tiempos de aplicación más adecuados para potenciar la atención en diferentes grupos etarios.

La realización de AF en la escuela está muy limitada hoy en día (Mahar et al., 2006). El tiempo dedicado a EF tanto en Educación Primaria como en Educación Secundaria es escaso. El profesorado se queja diariamente de la falta de atención y concentración que el alumnado muestra en las aulas. Mahar et al., (2006) llegaron a la conclusión de que largos períodos de tiempo de instrucción sin interrupción podrían ser contraproducentes para el rendimiento académico señalando que los niños que se someten a períodos prolongados de instrucción académica, a menudo, se vuelven más inquietos o intranquilos, llevándolos a una disminución de la concentración y la atención. En consecuencia, los déficits en la atención se asocian con un peor rendimiento académico (Aronen, Vuontela, Steenari, Salmi, \& Carlson, 2005; Jarrett \& Maxwell, 1998;Pellegrini, Huberty, \& Jones, 1995). No puede ser ignorado el hecho de que la práctica de AF principalmente en la etapa preadolescente, motiva al alumnado hacia la tarea ejerciendo un efecto positivo en el desarrollo cognitivo (GómezLópez et al., 2015). Asimismo, la capacidad atencional es clave para un correcto funcionamiento cognitivo siempre y cuando sea evaluado con técnicas adecuadas y meticulosamente elaboradas (Ison \& Carrada, 1995).

Teniendo en cuenta la anterior información, el objetivo de este estudio fue evaluar el efecto agudo de dos tipos de clases de EF, caracterizadas por intensidades de ejercicio y demandas cognitivas y sociales diferentes en la atención selectiva y la concentración en estudiantes de Educación Secundaria.

\section{Método}

\section{Participantes}

Los participantes fueron 98 estudiantes, 47 niñas (edad $=12.62 \pm 0.67$ años) y 51 niños (edad $=12.92 \pm 0.96$ años) de Educación Secundaria, que pertenecen a los cursos de $1^{\circ}$ y $2^{\circ}$ de un instituto del sur de España. Los estudiantes con discapacidad intelectual o física fueron excluidos de la misma. El estudio se realizó en cumplimiento de las normas de la Declaración de Helsinki (versión del 2013) y siguiendo las directrices de la Comunidad Europea de Buenas Prácticas Clínicas (111/3976/88 de julio de 1990), así como el marco legal español para la investigación clínica en seres humanos (Real Decreto 561/1993 sobre ensayos clínicos).De igual forma, el estudio fue aprobado por el Comité de Bioética de la Universidad de Jaén. Los estudiantes fueron asignados aleatoriamente mediante un muestro aleatorio simple en dos grupos experimentales, uno de juegos predeportivos (JPD $n=33$ ) y otro de juegos aeróbicos (JA n=32) y en un grupo control $(\mathrm{GCn}=33)$.

\section{Instrumentos y medidas}

Se utilizó el test D2 para la evaluación de la atención selectiva y la concentración (Brickenkamp, 2002) en su versión española (Seisdedos, 1997). Esta prueba determina la capacidad para focalizarse en un estímulo mientras se suprime de forma consciente ciertos distractores. Consta de 14 líneas de 47 letras mezcladas al azar (d o p) y se le pide a los sujetos que distinguiesen lo más rápido posible (dentro de los 20 segundos permitidos para cada línea) sólo la letra "d" con dos comillas (") que pueden aparecer ya sea por encima, por debajo o por separado, con una marca (') que aparecen arriba y abajo. Las letras 'p' son los distractores (Izquierdo et al., 2007). La prueba dura en total 280 segundos. Las variables analizadas fueron: respuesta total (TR), respuestas aceptadas (RA), el total de errores de comisión (C), el total de errores de omisión (O), eficacia global de la prueba (TOT) y la concentración (CON) (Jiménez et al., 2015). La fiabilidad es justificada a través de la consistencia interna de la prueba obteniendo un Alpha de Cronbach para la concentración de 0,96 
para niños (Culbertson \& Zillmer, 1998). El D2 tiene una validez convergente que permite observar una correlación significativa en concentración con el Trail Making $A=.33$ y el Trail Making $\mathrm{B}=.36$; el test $\mathrm{SDMT}=.47$; y el test STROOP=.34 (Culbertson \& Zillmer, 1998). La validez de constructo se determina mediante un análisis factorial donde un factor $1^{\circ}$ se denomina atención selectiva definiéndose principalmente con las variables TR, CON y VAR (índice de variación) del $\mathrm{d} 2$. Por otro lado, un $2^{\circ}$ factor denominado fexibilidad mental definido principalmente por el índice de precisión (E\%) del d2.

Para la evaluación del esfuerzo percibido (RPE) después de la finalización de la sesión de JPD y JA, se utilizó la Escala de Borg (Borg, 1982) cuyo elementos van de 0-10 desde una intensidad baja a alta intensidad del esfuerzo.

\section{Procedimiento}

En primer lugar, se obtuvo el consentimiento informado de los padres para la participación del alumnado en la investigación. A continuación, los tres grupos de alumnos (JPD, JA y GC) cumplimentaron el test D2 para recoger los datos del pre-test en su grupo de clase y en presencia del profesor. El test fue suministrado por un investigador con experiencia y entrenado en el uso del D2. Una semana más tarde, los grupos experimentales realizaron dos tipos de sesiones de EF una de JPD y otra de JA. Los JPD tienen una vinculación con los deportes, más allá de la mera realización de una actividad aeróbica, así el grupo JPD ejecutaba diferentes juegos en los que se llevaba a cabo una participación grupal del alumnado, requiriendo la cooperación con otros niños, en los que se producía cierto comportamiento estratégico (relaciones de cooperación y oposición), coordinación y adaptación a la cambiante demanda de la tarea continua (ejemplos: juegos de pase e interceptación de balón). Mientras que los participantes del grupo de JA se centraban en realizar ejercicios aeróbicos que requerían movimientos sencillos y generales sin manejo de móviles y sin establecerse relaciones de cooperación y oposición entre los participantes. Justo al finalizar la clase de EF, los alumnos tuvieron que valorar su RPE. Seguidamente, los grupos experimentales, volvieron a ejecutar el test D2 en la hora de clase inmediatamente posterior, al igual que el GC que no había recibido ninguna clase de EF a lo largo del día.

\section{Análisis estadístico}

Los resultados se muestran en estadísticos descriptivos como la media y desviación típica (DT). Se realizaron pruebas de distribución normal y homogeneidad (Kolmogorov-Smirnov \& Levene respectivamente) en todos los datos antes del análisis. Se realizó un análisis de varianza (ANOVA) entre los grupos experimentales en el pretest, postest y el postest-pretest, con un análisis post hoc mediante ajuste Bonferroni. El nivel de significación fue $p<.05$. El análisis de los datos se realizó utilizando el programa estadístico SPSS (versión 21, SPSS Inc., Chicago, IL).

\section{Resultados}

La Tabla 1 muestra los resultados obtenidos en el test D2 en los tres grupos estudiados. No se encuentran diferencias significativas en ninguna variable entre los tres grupos durante el pretest. En el postest, el grupo de JA obtiene mejor puntuación en concentración y total de aciertos con diferencias significativas $(p<.05)$ con el GC. En los resultados postest-pretest, el JPD obtiene los mejores resultados en concentración, total de aciertos y total de respuestas con diferencias significativas $(p<.05)$ en relación con el GC. Los grupos de JPD y JA obtienen resultados similares en la puntuación total con diferencias significativas $(p<.05)$ con el GC. Por último, el grupo de JA consigue la mejor puntuación en errores por omisión con diferencias significativas $(p<.05)$ con el GC. Finalmente, se encuentran diferencias significativas $(p<.001)$ en RPE entre los grupos experimentales, alcanzando el grupo de JA mayor RPE que el grupo de JPD (6.94 \pm 2.18 vs. $4.24 \pm 2.07, p<.05)$. 
Tabla 1. Resultados del test D2 durante pretest, postest y postest-pretest en los grupos analizados

\begin{tabular}{|c|c|c|c|c|c|c|c|c|c|}
\hline & & $\begin{array}{l}\text { Pretest } \\
\text { Media } \\
\text { (DT) }\end{array}$ & $\begin{array}{c}p- \\
\text { valor }\end{array}$ & $\begin{array}{l}\text { Postest } \\
\text { Media } \\
\text { (DT) }\end{array}$ & $\begin{array}{c}p- \\
\text { valor }\end{array}$ & $\begin{array}{l}\text { Post hoc } \\
\text { análisis }\end{array}$ & $\begin{array}{l}\text { Postes-pretest } \\
\text { Media (DT) }\end{array}$ & $\begin{array}{c}p- \\
\text { valor }\end{array}$ & $\begin{array}{l}\text { Post hoc } \\
\text { análisis }\end{array}$ \\
\hline \multirow{3}{*}{ Concentración } & JPD & $\begin{array}{l}147.24 \\
(44.05)\end{array}$ & .136 & $\begin{array}{l}179.09 \\
(47.39)\end{array}$ & .032 & $\mathrm{JA}>\mathrm{GC}^{*}$ & 31.84 (39.70) & .015 & JPD>GC* \\
\hline & JA & $\begin{array}{l}167.88 \\
(45.97)\end{array}$ & & $\begin{array}{l}196.81 \\
(49.55)\end{array}$ & & & $28.93(36.40)$ & & \\
\hline & GC & $\begin{array}{l}157.88 \\
(32.45)\end{array}$ & & $\begin{array}{l}165.88 \\
(43.56)\end{array}$ & & & $8.00(30.30)$ & & \\
\hline \multirow{3}{*}{$\begin{array}{l}\text { Total } \\
\text { puntuación }\end{array}$} & JPD & $\begin{array}{l}131.30 \\
(46.13)\end{array}$ & .410 & $\begin{array}{l}166.76 \\
(52.64)\end{array}$ & .116 & & $\begin{array}{c}35.45 \\
(39.74)\end{array}$ & .017 & $\begin{array}{c}\mathrm{JPD}>\mathrm{GC}^{*} \\
\mathrm{JA}>\mathrm{GC}^{*}\end{array}$ \\
\hline & JA & $\begin{array}{l}146.03 \\
(59.99)\end{array}$ & & $\begin{array}{l}182.63 \\
(54.46)\end{array}$ & & & $36.59(46.01)$ & & \\
\hline & GC & $\begin{array}{l}144.61 \\
(38.96)\end{array}$ & & $\begin{array}{l}156.27 \\
(45.17)\end{array}$ & & & $11.66(30.13)$ & & \\
\hline \multirow{3}{*}{ Total aciertos } & JPD & $\begin{array}{l}151.67 \\
(41.55)\end{array}$ & .133 & $\begin{array}{l}183.27 \\
(44.46)\end{array}$ & 0.031 & $\mathrm{JA}>\mathrm{GC}^{*}$ & $31.60(38.01)$ & .018 & JPD>GC* \\
\hline & JA & $\begin{array}{l}171.72 \\
(45.91)\end{array}$ & & $\begin{array}{l}198.34 \\
(49.64)\end{array}$ & & & $26.62(36.83)$ & & \\
\hline & GC & $\begin{array}{l}160.21 \\
(31.14)\end{array}$ & & $\begin{array}{l}167.42 \\
(44.96)\end{array}$ & & & $7.21(33.45)$ & & \\
\hline \multirow{3}{*}{$\begin{array}{l}\text { Errores } \\
\text { omisión }\end{array}$} & JPD & $\begin{array}{c}20.42 \\
(14.30)\end{array}$ & .155 & $\begin{array}{c}15.18 \\
(13.02)\end{array}$ & .652 & & $-5.24(10.74)$ & .024 & $\mathrm{JA}>\mathrm{GC}^{*}$ \\
\hline & JA & $\begin{array}{c}24.50 \\
(32.19)\end{array}$ & & $\begin{array}{c}12.56 \\
(11.92)\end{array}$ & & & $-11.93(23.82)$ & & \\
\hline & GC & $\begin{array}{c}14.18 \\
(12.78)\end{array}$ & & $\begin{array}{l}13.48 \\
(9.53)\end{array}$ & & & $-.69(11.47)$ & & \\
\hline \multirow{3}{*}{$\begin{array}{l}\text { Errores por } \\
\text { comisión }\end{array}$} & JPD & $\begin{array}{c}4.18 \\
(6.19)\end{array}$ & 0.134 & $\begin{array}{l}4.21 \\
(7.61)\end{array}$ & .038 & & $.03(7.74)$ & .203 & \\
\hline & JA & $\begin{array}{c}3.84 \\
(6.32)\end{array}$ & & $\begin{array}{c}1.59 \\
(1.82)\end{array}$ & & & $-2.25(5.61)$ & & \\
\hline & GC & $\begin{array}{c}1.79 \\
(1.63)\end{array}$ & & $\begin{array}{c}1.61 \\
(2.06)\end{array}$ & & & $-.18(1.82)$ & & \\
\hline \multirow{3}{*}{$\begin{array}{l}\text { Total de } \\
\text { respuestas }\end{array}$} & JPD & $\begin{array}{l}176.21 \\
(41.48)\end{array}$ & 0.064 & $\begin{array}{l}202.64 \\
(38.82)\end{array}$ & & & $31.66(38.00)$ & .046 & \\
\hline & JA & $\begin{array}{l}196.69 \\
(48.63)\end{array}$ & & $\begin{array}{l}203.16 \\
(55.42)\end{array}$ & 0.217 & & $24.25(38.20)$ & & $\mathrm{JPD}>\mathrm{GC}^{*}$ \\
\hline & GC & $\begin{array}{l}175.88 \\
(28.54)\end{array}$ & & $\begin{array}{l}185.48 \\
(43.09)\end{array}$ & & & $10.33(27.46)$ & & \\
\hline
\end{tabular}

Nota. JPD= Juegos predeportivos; JA= Juegos aeróbicos; GC $=$ Grupo control. * $p<.05 . \mathrm{DT}=$ desviación típica.

\section{Discusión}

El objetivo de este estudio fue analizar el efecto de dos tipos de clases de EF basadas en JA y JPD en la atención selectiva y la concentración de estudiantes de secundaria. El principal hallazgo de este estudio es que los niños que recibieron una clase de EF obtienen mejores puntuaciones en las distintas variables del test D2 en relación con el grupo que no hizo EF en esa jornada escolar. El grupo de JA manifiesta mayor intensidad percibida durante la clase de EF, sin embargo el grupo de JPD experimenta un mayor incremento en los valores finales de concentración en relación con el GC.

Las actividades realizadas en la sesión de JA pueden producir, según afirma Yanagisawa et al., (2010), una activación de las corteza prefrontal dorsolateral izquierda que son también responsables de funciones cognitivas como la atención. Hillman, Buck, Themanson, Pontifex, \&
Castelli, (2009) mostraron que los periodos cortos de ejercicio aeróbico moderado-intenso pueden mejorar el control cognitivo de la atención en los preadolescentes. Este hecho puede ser debido también a la relación entre el ejercicio, las catecolaminas, la concentración y la cognición (McMorris, Sproule, Turner, \& Hale, 2011). A su vez, Pontifex et al., (2013) mostraron un mayor rendimiento en las áreas de lectura y aritmética después de una sola sesión de 20 minutos de ejercicio físico en niños de entre 8-10 años de edad. Además, Budde et al., (2008) encontraron mejoras significativas en la atención y la concentración utilizando la prueba D2 en los adolescentes después de una clase de EF de ejercicios de coordinación frente a una clase de EF basada en la realización de deportes colectivos. Por otro lado, Pirrie \& Lodewyk (2012) analizaron la influencia de la AF de moderada a vigorosa en cuatro procesos cognitivos, como la planificación, la atención, el 
procesamiento simultáneo y procesamiento sucesivo; y los resultados indicaron que el rendimiento en la prueba de planificación mejoró significativamente después de la $\mathrm{AF}$, aunque no se observó mejoría de la atención, el procesamiento simultáneo o sucesivo de procesamiento. Estos resultados podrían deberse a que el tipo de $\mathrm{AF}$, su intensidad y duración podrían tener influencia en el efecto agudo de una sesión de AF sobre la atención (Janssen et al., 2014).

Es importante tener en cuenta que los ejercicios aeróbicos repetitivos probablemente requieren menos participación cognitiva, particularmente en funciones ejecutivas ya que hay poca necesidad de guiar la cognición para lograr una meta difícil o coordinar el cuerpo para ejecutar movimientos complejos (Best, 2010). Estas diferencias en las demandas de la ejecución de las funciones ejecutivas, pueden conducir a que un ejercicio más complejo, como los JPD empleados en este estudio, provoquen mejoras más importantes en la concentración. Sería adecuado que diferentes formas de $\mathrm{AF}$ se probaran en la población escolar para determinar si éstas tienen efectos importantes y diferenciadores en el rendimiento cognitivo (Janssen et al., 2014).

Este estudio tiene ciertas limitaciones como la dificultad de determinar en una sola prueba los niveles atencionales ya que este aspecto de la cognición humana es relativamente susceptible a ser cambiante por diversos motivos externos (Wang, Chu, Chu, Chan, \& Chang, 2013). A su vez, otra limitación es que no se ha mostrado el efecto diferenciador en relación con el sexo de los participantes. Sin embargo, dispone de muchos puntos fuertes, entre ellos, que dicho estudio se ha llevado a cabo en el contexto escolar y no en un laboratorio como muchos estudios previos (Hillman et al., 2009; Pontifex et al., 2013). A su vez, este estudio pone a prueba dos estrategias diferentes para corroborar la asociación entre la AF y la atención, una actividad aeróbica de mayor intensidad con escasa interacción social y exigencias cognitivas (JA) y otra AF con menor intensidad pero mayor socialización y exigencia táctica (JPD). Como posibles estudios futuros se plantea nuevas investigaciones que abarquen las limitaciones comparativas por sexo así como analizar otras edades. Del mismo modo, pueden ser atendidos otros tipos de estímulos iniciales para determinar la mejora o no en la atención.

En conclusión, los resultados obtenidos en este estudio muestran que la ejecución de ejercicios aeróbicos moderados, socializantes y que impliquen estrategia como los JPD, provocan un efecto agudo en la mejora de la atención selectiva en los niños durante el día escolar. Los resultados indican la importancia de utilizar la $\mathrm{AF}$ como medio de regulación de la atención en el aula entre los jóvenes. En este sentido, la literatura sugiere que el rendimiento académico, la condición física y la salud de los estudiantes no se mejora mediante la limitación del tiempo asignado a la EF y la AF (Trudeau \& Shephard, 2008). Por tanto, el aumento de la cantidad de tiempo dedicado a la EF puede promover beneficios cognitivos agudos, tales como la mejora de la atención y la concentración, así como un aumento de la motivación del alumnado (Sánchez-Oliva, Leo Marcos, Amado Alonso, Pulido-González, \& García-Calvo, 2015; Gómez-López et al., 2015) que tienen importantes implicaciones en la escuela.

\section{Referencias}

Ahn, S., \& Fedewa, A. L. (2011). A meta-analysis of the relationship between children's physical activity and mental health. Journal of Pediatric Psychology, 36(4), 385-97. doi:http://doi.org/10.1093/jpepsy/jsq107

Altenburg, T. M., Chinapaw, M. J. M., \& Singh, A. S. (2016). Effects of one versus two bouts of moderate intensity physical activity on selective attention during a school morning in Dutch primary schoolchildren: A randomized controlled trial. Journal of Science and Medicine in Sport, 19(10), 820-824.

doi:http://doi.org/10.1016/j.jsams.2015.12.003

Aronen, E. T., Vuontela, V., Steenari, M.R., Salmi, J., \& Carlson, S. (2005). Working memory, psychiatric symptoms, and academic performance at school. Neurobiology of Learning and Memory, 83(1), 33-42. doi: http://doi.org/10.1016/j.nlm.2004.06.010

Best, J. R. (2010). Effects of physical activity on children's executive function: Contributions of experimental research on aerobic exercise. Developmental Review, 30(4), 331-351. 
doi:http://doi.org/10.1016/j.dr.2010.08.001

Borg, G. (1982). Psychophysical bases of perceived exertion. Medicine and Science in Sports and Exercise, 14(5), 377-381.

Brickenkamp, R. (2002). Test d2: Aufmerksamkeits-Belastungs-Test; Manual. Gottingen: Hogrefe

Budde, H., Voelcker-Rehage, C., PietraßykKendziorra, S., Ribeiro, P., \& Tidow, G. (2008). Acute coordinative exercise improves attentional performance in adolescents. Neuroscience Letters, 441(2), 219-223. doi:http://doi.org/10.1016/j.neulet.2008.06.024

Chaddock, L., Pontifex, M. B., Hillman, C. H., \& Kramer, A. F. (2011). A review of the relation of aerobic fitness and physical activity to brain structure and function in children. Journal of the International Neuropsychological Society: JINS, 17(6), 975-85. doi:http://doi.org/10.1017/S1355617711000567

Culbertson, W. C., \& Zillmer, E. A. (1998). The Tower of London(DX): A standardized approach to assessing executive functioning in children. Archives of Clinical Neuropsychology: The Official Journal of the National Academy of Neuropsychologists, 13(3), 285-301.

Delgado, B. G., \& Prado-Gascón, V. J. (2016). Relación entre la práctica de actividad física y el rendimiento académico en escolares adolescentes. Calidad de Vida Y Salud, 9(1), 60-73.

Dutke, S., Jaitner, T., Berse, T., \& Barenberg, J. (2014). Acute Physical Exercise Affected Processing Efficiency in an Auditory Attention Task More than Processing Effectiveness. Journal of Sport and Exercise Psychology, 36(1), 69-79. doi:http://doi.org/10.1123/jsep.2013-0044

Ellemberg, D., \& St-Louis-Deschênes, M. (2010). The effect of acute physical exercise on cognitive function during development. Psychology of Sport and Exercise, 11(2), 122126. doi: http//doi.org/10.1016/j.psychsport.2009.09.006 Gallotta, M. C., Emerenziani, G. P., Franciosi, E., Meucci, M., Guidetti, L., \& Baldari, C. (2015). Acute physical activity and delayed attention in primary school students. Scandinavian Journal of Medicine \& Science in Sports, 25(3), e331-e338.

doi:http://doi.org/10.1111/sms.12310

Gómez-López, M., Granero-Gallegos, A., BaenaExtremera, A., Amador, C. B., Javier, y F., \& Quero, P. (2015). Efectos de Sexo y Práctica de Ejercicio Físico sobre la Educación Física. Revista Iberoamericana de Diagnóstico $Y$ Evaluación - e Avaliação Psicológica, 40(2), 6-16.

Hillman, C. H., Buck, S. M., Themanson, J. R., Pontifex, M. B., \& Castelli, D. M. (2009). Aerobic fitness and cognitive development: Event-related brain potential and task performance indices of executive control in preadolescent children. Developmental Psychology, 45(1), 114-29.

doi:http://doi.org/10.1037/a0014437

Ison, M. S., \& Carrada, M. (1995). Evaluación de la eficacia atencional. Estudio normativo preliminar en escolares argentinos. Revista Iberoamericana de Diagnóstico y Evaluación - e Avaliação Psicológica, 1(29), 129-146.

Luliano, E., Di Cagno, A., Aquino, G., Fiorilli, G., Mignogna, P., Calcagno, G., \& Di Costanzo, A. (2015). Effects of different types of physical activity on the cognitive functions and attention in older people: A randomized controlled study. Experimental Gerontology, 70, 105-110. http://doi.org/10.1016/j.exger.2015.07.008

Izquierdo, M. C., De Iscar Pérez, M. J., Losa, M. A. B., López, M. M., Pérez, L. Á., Solís, G., Pérez, J. L. A. (2007). Psychometric properties of the $\mathrm{d} 2$ selective attention test in a sample of premature and born-at-term babies. Psicothema, 19(4), 706-710.

Janssen, I., \& Leblanc, A. G. (2010). Systematic review of the health benefits of physical activity and fitness in school-aged children and youth. International Journal of Behavioral Nutrition and Physical Activity, 7(40),1-16.

doi:http://doi.org/1479-5868-7-40

[pii]ไr10.1186/1479-5868-7-40

Janssen, M., Toussaint, H. M., van Mechelen, W., \& Verhagen, E. A. (2014). Effects of acute bouts of physical activity on children's attention: a systematic review of the literature. SpringerPlus, 3(410), 1-10.

doi:http://doi.org/10.1186/2193-1801-3-410

Jarrett, O., \& Maxwell, D. (1998). Impact of 
recess on classroom behavior: Group effects and individual differences. The Journal of Educational Research, 92(2), 121-126.

Jiménez, J. E., Jiménez, J. E., Hernández, S., García, E., Díaz, A., Rodríguez, C., \& Martín, R. (2015). Test de atención D2: Datos normativos y desarrollo evolutivo de la atención en educación primaria. European Journal of Education and Psychology, 5(1). doi:http://doi.org/10.1989/ejep.v5i1.93

Mahar, M. T., Murphy, S. K., Rowe, D. A., Golden, J., Shields, A. T., \& Raedeke, T. D. (2006). Effects of a classroom-based program on physical activity and on-task behavior. Medicine and Science in Sports and Exercise, 38(12), 2086-94.

doi:http//doi.org/10.1249/01.mss.0000235359.16685.a3

Maureira-Cid, F., \& Flores-Ferro, E. (2017). Efectos del ejercicio físico sobre la atención: Una revisión de los últimos años. Revista Ciencias de La Actividad Física, 18(1), 73-83

McMorris, T., Sproule, J., Turner, A., \& Hale, B. J. (2011). Acute, intermediate intensity exercise, and speed and accuracy in working memory tasks: a meta-analytical comparison of effects. Physiology \& Behavior, 102(3-4), 421-428.

doi:http://doi.org/10.1016/j.physbeh.2010.12.007

Pellegrini, A. D., Huberty, P. D., \& Jones, I. (1995). The effects of recess timing on children's playground and classroom behaviors. American Educational Research Journal, 32(4), 845-864. doi:http://doi.org/10.3102/00028312032004845

Pérez, L., Padilla, C., Parmentier, F. B. R., \& Andrés, P. (2014). The effects of chronic exercise on attentional networks. PloS One, 9(7), e101478. doi:http://doi.org/10.1371/journal.pone.0101478

Pesce, C., Crova, C., Cereatti, L., Casella, R., \& Bellucci, M. (2009). Physical activity and mental performance in preadolescents: Effects of acute exercise on free-recall memory. Mental Health and Physical Activity, 2, 16-22. doi:http://doi.org/10.1016/j.mhpa.2009.02.001

Pirrie, A. M., \& Lodewyk, K. R. (2012). Investigating links between moderate-tovigorous physical activity and cognitive performance in elementary school students. Mental Health and Physical Activity, 5(1), 93-
98. doi:http://doi.org/10.1016/j.mhpa.2012.04.001

Pontifex, M. B., Hillman, C. H., Fernhall, B., Thompson, K. M., \& Valentini, T. a. (2009). The effect of acute aerobic and resistance exercise on working memory. Medicine and Science in Sports and Exercise, 41(4), 927934.

doi:http://doi.org/10.1249/MSS.0b013e3181907d69 Pontifex, M., Saliba, B., Raine, L., \& Picchietti, D. (2013). El ejercicio mejora del comportamiento, neurocognitivo y rendimiento escolar en niños con déficit de atención/hiperactividad. El Diario Deportivo, 162(3), 543-551.

Sánchez-Oliva, D., Leo Marcos, F. M., Amado Alonso, D., Pulido-González, J. J., \& GarcíaCalvo, T. (2015). Análisis de los perfiles motivacionales y su relación con los comportamientos adaptativos en las clases de educación física. Revista Latinoamericana de Psicología, 47(3), 156-166. doi:http://doi.org/10.1016/j.rlp.2015.06.007

Schmidt, M., Egger, F., \& Conzelmann, A. (2015). Delayed Positive Effects of an Acute Bout of Coordinative Exercise on Children's Attention. Perceptual and Motor Skills, 121(2), 431-446.

doi:http://doi.org/10.2466/22.06.PMS.121c22x1

Seisdedos, N. (1997). Adaptación y creación de recursos psicológicos. En Psicología del trabajo y Gestión de Recursos Humanos, obra coordinada por M. Ordoñez. Barcelona: Grao.

Soga, K., Shishido, T., \& Nagatomi, R. (2015). Executive function during and after acute moderate aerobic exercise in adolescents. Psychology of Sport and Exercise, 16, 7-17. doi:http://doi.org/10.1016/j.psychsport.2014.08.010

Tomporowski, P. D. (2003). Effects of acute bouts of exercise on cognition. Acta Psychologica, 112(3), 297-324.

doi:http://doi.org/10.1016/S0001-6918(02)00134-8

Tomporowski, P. D., Davis, C. L., Miller, P. H., \& Naglieri, J. a. (2008). Exercise and children's intelligence, cognition, and academic achievement. Educational Psychology Review, 20(2), 111-131. doi:http://doi.org/10.1007/s10648-007-9057-0

Trudeau, F., \& Shephard, R. J. (2008). Physical education, school physical activity, school sports and academic performance. 
International Journal of Behavioral Nutrition and Physical Activity. doi:http://doi.org/10.1186/1479-5868-5-10

Verburgh, L., Königs, M., Scherder, E. J. a, \& Oosterlaan, J. (2014). Physical exercise and executive functions in preadolescent children, adolescents and young adults: a meta-analysis. British Journal of Sports Medicine, 48(12), 973-9. doi:http://doi.org/10.1136/bjsports2012-091441

Vidarte-Claros, J. A., Velez-Álvarez, C., Sandoval-Cuellar, C., \& Alfonso-Mora, M. lorena. (2011). Actividad física: Estrategia de promoción de la salud. Hacia La Promoción de La Salud, 16(1), 202-218.

Vidoni, E. D., Johnson, D. K., Morris, J. K., Van Sciver, A., Greer, C. S., Billinger, S. A., \& Burns, J. M. (2015). Dose-response of aerobic exercise on cognition: A community-based, pilot randomized controlled trial. PloS One, 10(7), e0131647. doi:http://doi.org/10.1371/journal.pone.0131647

Wang, C. C., Chu, C. H., Chu, I. H., Chan, K. H., \& Chang, Y. K. (2013). Executive function during acute exercise: The role of exercise intensity. Journal of Sport \& Exercise Psychology, 35(4), 358-67.

Wilson, A. N., Olds, T., Lushington, K., Petkov, J., \& Dollman, J. (2016). The impact of 10minute activity breaks outside the classroom on male students' on-task behaviour and sustained attention: A randomised crossover design. Acta Paediatrica, 105(4), e181-e188. doi:http://doi.org/10.1111/apa.13323

Yanagisawa, H., Dan, I., Tsuzuki, D., Kato, M., Okamoto, M., Kyutoku, Y., \& Soya, H. (2010). Acute moderate exercise elicits increased dorsolateral prefrontal activation and improves cognitive performance with Stroop test. NeuroImage, 50(4), 1702-1710. doi:http://doi.org/10.1016/j.neuroimage.2009.12.023 